\title{
Programming with Permissions in Mezzo
}

\author{
François Pottier \\ INRIA \\ francois.pottier@inria.fr
}

\author{
Jonathan Protzenko \\ INRIA \\ jonathan.protzenko@ens-lyon.org
}

\begin{abstract}
We present $M e z z o$, a typed programming language of ML lineage. Mezzo is equipped with a novel static discipline of duplicable and affine permissions, which controls aliasing and ownership. This rules out certain mistakes, including representation exposure and data races, and enables new idioms, such as gradual initialization, memory re-use, and (type)state changes. Although the core static discipline disallows sharing a mutable data structure, $M e z^{z} O$ offers several ways of working around this restriction, including a novel dynamic ownership control mechanism which we dub "adoption and abandon".
\end{abstract}

Categories and Subject Descriptors D.3.2 [Programming Languages]: Language Classifications-Multiparadigm languages

Keywords static type systems; side effects; aliasing; ownership

\section{Introduction}

Programming with mutable, heap-allocated data structures is hard. In many typed imperative programming languages, including Java, C\#, and ML, the type system keeps track of the structure of objects, but not of how they are aliased. As a result, a programming mistake can cause undesired sharing, which in turn leads to breaches of abstraction, invariant violations, race conditions, and so on. Furthermore, the fact that sharing is uncontrolled implies that the type of an object must never change. This forbids certain idioms, such as delayed initialization, and prevents the type system from keeping track of the manner in which objects change state through method calls. In order to work around this limitation, programmers typically use C\# and Java's null pointer, or ML's option type. This implies that a failure to follow an intended protocol is not detected at compile time, but leads to a runtime error. In short, there is a price to pay for the simplicity of traditional type systems: the bugs caused by undesired sharing, or by the failure to follow an object protocol, are not statically detected.

This paper presents the design of a new programming language, Mezzo, which attempts to address these issues. One motivating principle behind the design of Mezzo is that one should be able to express precise assertions about the current state of an object or data structure. The type system should keep track of state changes and forbid using an object in a manner that is inconsistent with

Permission to make digital or hard copies of all or part of this work for personal or classroom use is granted without fee provided that copies are not made or distributed for profit or commercial advantage and that copies bear this notice and the full citation on the first page. Copyrights for components of this work owned by others than the author(s) must be honored. Abstracting with credit is permitted. To copy otherwise, or republish, to post on servers or to redistribute to lists, requires prior specific permission and/or a fee. Request permissions from permissions@ acm.org.

ICFP '13, September 25-27, 2013, Boston, MA, USA.

Copyright is held by the owner/author(s). Publication rights licensed to ACM. ACM 978-1-4503-2326-0/13/09...\$15.00.

http://dx.doi.org/10.1145/2500365.2500598 its current state. An example is a socket that moves from state "ready", to "connected", then to "closed". The "close" function, for instance, should be invoked only if the socket is currently in the state "connected", and changes its state to "closed". Another example is a collection, which must not be accessed while an iterator exists, but can be used again once iteration is over.

Although state and state change play an important role in many programs, no mainstream programming language builds these notions into its static discipline. External tools must be used, such as typestate checking tools [13, 5, 6] or tools for constructing proofs of programs, based for instance on separation logic [4, 20] or on the Spec\# methodology [3]. Instead, we explore the possibility of reasoning about state within the type system. This has well-known potential benefits. A property that is expressed as a type is checked early, often, and at little cost. Furthermore, we believe that, in the future, such a type system can serve as a strong foundation for performing proofs of programs.

Obviously, if two "principals" separately think that "the socket $s$ is currently connected", and if one of them decides to close this socket, then the other will be left with an incorrect belief about $s$. Thus, precise reasoning about state and state changes requires that information about a mutable object (or data structure) be recorded in at most "one place" in the type system. In Mezzo, this place is a permission. Permissions keep track not only of the structure of data, as does a traditional type system, but also of must-alias and must-not-alias (i.e. equality and disjointness) information. Like a separation logic assertion [29], a permission has an ownership reading: to have access to a description of a part of the heap is to own this part of the heap. Because "to describe is to own", we need not explicitly annotate types with owners, as done in Ownership Types [10] or Universe Types [14].

We do not think of the "type" of an object and of its "state" as two distinct notions: a permission describes both at once. Whereas previous work on permissions [5] distinguishes between a fixed type structure and "permissions" that evolve with time, in Mezzo, both "type" and "state" can change over time. This yields greater expressiveness: for instance, gradual initialization and memory reuse become possible. This also yields greater simplicity and conciseness: for instance, when we write polymorphic code that manipulates a list, a single type variable $a$ denotes not only "what" the list elements are (e.g., sockets) but also in what "state" they are and to what extent we "own" them.

The choices described above form our basic design premises. Mezzo can be viewed as an experiment, whose aim is to determine to what extent these choices are viable. Beyond these decisions, we strive to make the language as simple as possible. Mezzo is a high-level programming language: we equip it with first-class functions, algebraic data types, and require a garbage collector. We could have chosen classes and objects instead of (or in addition to) algebraic data types; this could be a topic for future research. We equip Mezzo with a simple distinction between duplicable permissions (for immutable data) and exclusive permissions (for 
mutable data). Although more advanced varieties of permissions exist in the literature, including read-only views of mutable data and fractional permissions [7], we wish to evaluate how far one can go without these advanced notions; if desired, they could in principle be added to $M e z z o$.

By default, Mezzo's permission discipline imposes a restrictive aliasing regime: the mutable part of the heap must form a forest. Mezzo offers several mechanisms for evading this restriction. One, adoption and abandon, is new. It allows arbitrary aliasing patterns within a region of the heap and achieves soundness via dynamic checks. We describe it in detail in \$7 The second mechanism is Boyland's nesting [7]. It can be viewed as a form of adoption and abandon that requires no runtime checks but is (for many purposes) less powerful. The last mechanism is locks in the style of concurrent separation logic [25, 18, 19, 8].

Mezzo's static discipline has been formally defined and mechanically proved sound The formalization, which is available online [26], includes adoption and abandon, but does not (at present) cover nesting, locks, or concurrency. The statement of soundness guarantees that "well-typed programs do not go wrong", except possibly when the dynamic check performed at "abandon" fails. In a concurrent extension of $M e z z o$, it would in addition guarantee that "well-typed programs are data-race-free". The proof is syntactic. We extend the typing judgement to a pair of a program under execution and its heap, and establish the standard subject reduction and progress lemmas.

A prototype type-checker has been implemented and is publicly available [27]. Several small libraries, totaling a few thousand lines of code, have been written, and are also available online [27]. They include immutable data structures (lists), mutable data structures (lists, doubly-linked lists, binary search trees, hash tables, resizable arrays, and FIFO queues, see \$7, persistent data structures implemented via imperative means (suspensions, persistent arrays), and a few algorithms (memoization; graph search). At the time of this writing, an interpreter is available, and a simple compiler (which translates Mezzo down to untyped OCaml) is being developed.

The paper begins with a motivating example ( $\$ 2$ ), which cannot be type-checked in ML, and which serves to informally illustrate Mezzo's permission discipline. Then, we define the syntax of types, permissions, and expressions ( $\$ 3$ ) and informally explain the ownership reading of permissions for immutable and mutable data $(\$ 4)$. We present the typing rules $(\$ 5$ ) and introduce a few syntactic conventions that make the surface language more palatable $(\$ 6$. We explain adoption and abandon, illustrate them with a second example (\$7), and discuss nesting and locks more briefly (\$8). Finally, we explain where $M e z z o$ lies in the design space and compare it with some of the previous approaches found in the literature $(\$ 9)$.

\section{Mezzo by example}

Figure 1 presents code for the concatenation of two immutable lists. This example showcases several of Mezzo's features, and allows us to explain the use of permissions. We review the code first $\$ 2.1$, then briefly explain how it is type-checked $(\$ 2.2$ and $\$ 2.3$.

\subsection{Code}

Our purpose is to write code that concatenates two immutable lists xs and ys to produce a new immutable list. The traditional, purely functional implementations of concatenation have linear space overhead, as they implicitly or explicitly allocate a reversed

\footnotetext{
${ }^{1}$ The formalization concerns a slightly lower-level language, Core Mezzo, In Core Mezzo, fields are numbered, whereas in Mezzo they are named and field names can be overloaded. At present, Core Mezzo is missing some of the features of Mezzo, including parameterized algebraic data types and mode constraints. We hope to add them in the future.
}

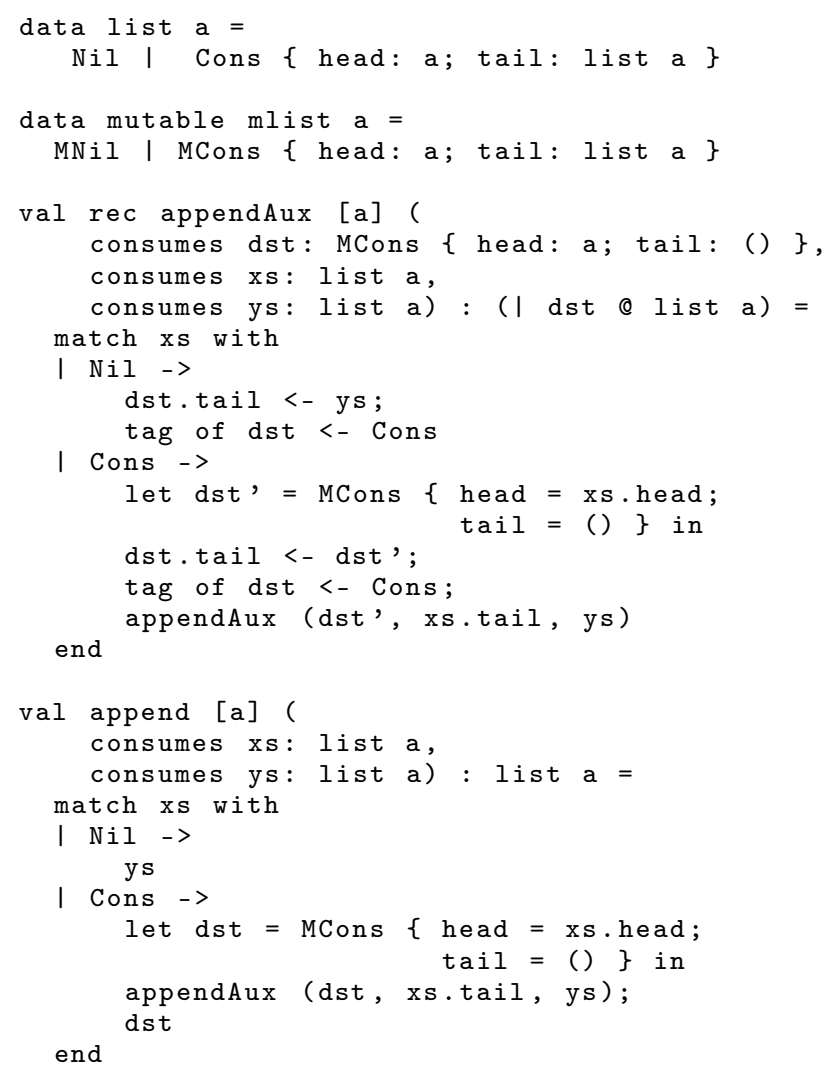

Figure 1. Tail-recursive concatenation of immutable lists

copy of xs. Our implementation, on the other hand, is written in destination-passing style, and has constant space overhead. Roughly speaking, the list xs is traversed and copied on the fly. When the end of xs is reached, the last cell of the copy is made to point to ys.

The append function (Figure 1 line 23) is where concatenation begins. If xs is empty, then the concatenation of $x s$ and ys is ys (line 27). Otherwise (line 29), append allocates an unfinished, mutable cell dst (line 30. This cell contains the first element of the final list, namely xs . head. It is in an intermediate state: it cannot be considered a valid list, since its tail field contains the unit value (). It is now up to appendAux to finish the work by constructing the concatenation of xs.tail and ys and writing the address of that list into dst.tail. Once appendAux returns, dst has become a wellformed list (this is indicated by the postcondition "dst @ list a" on line 10 and is returned by append.

The function appendAux expects an unfinished, mutable cell dst and two lists xs and ys. Its purpose is to write the concatenation of xs and ys into dst.tail, at which point dst can be considered a well-formed list. If xs is $\mathrm{Nil}$ (line 12), the tail field of dst is made to point to ys. Then, dst, a mutable MCons cell, is "frozen" by a tag update instruction and becomes an immutable Cons cell. (This instruction compiles to a no-op.) If xs is a Cons cell (line 15), we allocate a new destination cell dst', let dst.tail point to it, freeze $\mathrm{dst}$, and repeat the process via a tail-recursive call.

This example illustrates several important aspects of Mezzo.

Expressiveness In a traditional typed programming language, such as Java or OCaml, list concatenation in destination-passing style is possible, but its result must be a mutable list, because an immutable list cell cannot be gradually initialized. 
State change The call appendAux(dst, xs, ys) changes the "type" of dst from "unfinished, mutable list cell" to "well-formed, immutable list". This type-changing update is sound because one must be the "unique owner" of the mutable cell dst for this call to be permitted.

Ownership transfer In fact, the call appendAux(dst, xs, ys) also changes the "type" of xs and ys from "immutable list" to "unknown". Indeed, the postcondition of appendAux guarantees nothing about $x$ s and ys. In other words, the caller gives up the permission to use xs and ys as lists, and in return gains the permission to use dst as a list. In other words, the ownership of the list elements is transferred from xs and ys to dst. This is required for soundness. We do not know what the list elements are (they have abstract type a). They could be mutable objects, whose "unique ownership" property must not be violated.

\subsection{Permissions}

Permissions do not exist at runtime: they are purely an artefact of the type system. An atomic permission $x @ t$ represents the right to use the program variable $x$ at type $t$. Two permissions $P_{1}$ and $P_{2}$ can be combined to form a composite permission $P_{1} * P_{2}$. The conjunction $*$ is separating [29] at mutable memory locations and requires agreement at immutable locations $(4.1$. The empty permission, a unit for conjunction, is written empty.

When execution begins, a program conceptually possesses an empty permission. As execution progresses through the code, permissions come and go. At any program point, there is a certain current permission. Most of the time, the manner in which permissions evolve and flow is implicit. It must be made explicit in a few places: in particular, every function type must include explicit preand postconditions.

Let us continue our discussion of the concatenation example (Figure 11). We explain in an informal manner how the function append is type-checked. This allows us to illustrate how permissions are used and how they evolve.

The typing rules appear in Figure 4, a subset of the permission subsumption rules appear in Figure 6 In the following, we refer to some of these rules, but defer their detailed explanation to $\$ 5$

The append function is defined at line 23 At the beginning of the function's body, by the typing rule FUNCTION permissions for the formal arguments are available. Thus, the current permission is:

$$
x s @ \text { list } a * y s @ \text { list } a
$$

This permission represents the right to use xs and ys as lists of elements of type $a$.

This permission soon evolves, thanks to the match construct, which examines the tag carried by xs. By the typing rule MATCH as we learn that $\mathrm{xs}$ is a $\mathrm{Nil}$ cell, we replace our permission about xs with a more precise one, which incorporates the knowledge that the tag of xs is Nil. At line 27, the current permission becomes:

$$
x s @ \mathrm{Nil} * y s @ \text { list } a
$$

$x s @$ Nil is a structural permission: it asserts that xs points to a memory block whose tag is Nil (and which has zero fields). Similarly, at line 29 the current permission becomes:

$$
x s @ \text { Cons }\{\text { head : } a \text {; tail : list } a\} * y s @ \text { list } a
$$

The structural permission for xs asserts that xs points to a memory block that carries the tag Cons and has a head field of type $a$ and a tail field of type list $a$.

\footnotetext{
${ }^{2}$ We later note $(\sqrt{4.1}$ that if at a call site the variable a is instantiated with a duplicable type, say int, then the permissions xs @ list int and ys @ list int are considered duplicable, so they can in fact be duplicated prior to the call appendAux (dst, xs, ys), hence are not lost.
}

At this stage, the type-checker performs an implicit operation. It applies the permission subsumption rule DECOMPOSEBLOCK This causes fresh names $h d$ and $t l$ to be introduced for the head and tail fields of this structural permission. This yields the following conjunction:

$$
\begin{aligned}
& x s @ \text { Cons }\{\text { head : }(=h d) ; \text { tail : }(=t l)\} * \\
& h d @ a * t l @ \text { list } a * \\
& y s @ \text { list } a
\end{aligned}
$$

This is our first encounter of a singleton type, which we write $=h d$. A permission of the form $x @=y$ asserts that the variables $x$ and $y$ denote the same value. In particular, if they denote memory locations, this means that $x$ and $y$ point to the same object: this is a must-alias constraint. We write $x=y$ for $x @=y$. Similarly, in the structural permission above, the fact that the head field has type $=h d$ means that the value of this field is $h d$. We write head $=h d$ for head : $(=h d)$.

By the typing rules NEW and LET when the cell dst is allocated (line 30, a permission for dst appears, namely:

$$
d s t @ \text { MCons }\{\text { head }=h d ; \text { tail : () }\}
$$

We now see how singleton types help reason about sharing. At this point, we have three permissions that mention $h d$. We know that $h d$ is stored in the head field of $x s$; we know that $h d$ is stored in the head field of $d s t$; and we have a permission to use $h d$ at type $a$. We do not need a borrowing convention [24] in order to fix which of $x s$ or $d s t$ owns $h d$. Instead, the system knows that the object $h d$ is accessible via two paths, namely $x s$.head and $d s t$.head, and can be used under either name. This use of singleton types is taken from Alias Types [30].

By the typing rules READ and APPLICATION in order to call appendAux (dst, xs.tail, ys) (line 32), we need the following conjunction of permissions. It is the precondition of appendAux, suitably instantiated:

$$
d s t @ \text { MCons }\{\text { head : } a \text {; tail : () }\} * t l @ \text { list } a * y s @ \text { list } a
$$

Are we able to satisfy this requirement? The answer is positive. The subsumption rules EXISTSINTRO and DECOMPOSEBLOCK allow combining the permissions MCons $\{$ head $=h d$; tail : () $\}$ and $h d @ a$ (both of which are present) to obtain the first conjunct above. The second and third conjuncts above are present already.

By APPLICATION the precondition of appendAux is consumed (taken away from the caller). After the call, the postcondition of appendAux is added to the current permission, which is then:

$$
x s @ \text { Cons }\{\text { head }=h d ; \text { tail }=t l\} * d s t @ \text { list } a
$$

The conjunct that concerns $x s$ is of no use, and is in fact silently discarded when we reach the end of the Cons branch within append. The conjunct that concerns $d s t$ is used to check that this branch satisfies append's advertised return type, namely list $a$. Similarly, in the Nil branch, the permission ys @ list $a$ shows that a value of appropriate type is returned. In conclusion, append is well-typed.

\subsection{To loop or to tail call?}

In-place concatenation (that is, melding) of mutable lists can also be implemented by a tail-recursive function. The pattern is analogous to that of Figure 1 but the code is simpler, because the first list is not copied, and "freezing" is not required.

These algorithms are traditionally viewed as iterative and implemented using a while loop. Berdine et al.'s iterative formulation of mutable list melding [4], which is proved correct in separation logic, has a complex loop invariant, involving two "list segments", and requires an inductive proof that the concatenation of two list segments is a list segment. In contrast, in the tail-recursive approach, the "loop invariant" is the type of the recursive function 
(e.g., appendAux in Figure 1). This type is reasonably natural and does not involve list segments.

How do we get away without list segments and without inductive reasoning? The trick is that, even though appendAux is tailrecursive, which means that no code is executed after the call by appendAux to itself, a reasoning step still takes place after the call. Immediately before the call, the current permission can be written as follows:

$$
\begin{aligned}
& x s @ \text { Cons }\{\text { head }=h d ; \text { tail }=t l\} * \\
& d s t @ \text { Cons }\left\{\text { head : } a \text {; tail }=d s t^{\prime}\right\} * \\
& d s t^{\prime} @ \text { MCons }\{\text { head : } a \text {; tail : }()\} * \\
& t l @ \text { list } a * y s @ \text { list } a
\end{aligned}
$$

The call "appendAux (dst', xs.tail, ys)" consumes the last three permissions and produces instead $d s t^{\prime}$ @ list $a$. The first two permissions are "framed out", i.e., implicitly preserved. After the call, we have:

$$
\begin{array}{r}
x s @ \text { Cons }\{\text { head }=h d ; \text { tail }=t l\} * \\
d s t @ \text { Cons }\left\{\text { head : } a ; \text { tail }=d s t^{\prime}\right\} * \\
d s t^{\prime} @ \text { list } a
\end{array}
$$

Dropping the first permission and combining the last two yields:

$$
d s t @ \text { Cons }\{\text { head : } a \text {; tail : list } a\}
$$

which can be folded back to dst @ list $a$, so appendAux satisfies its postcondition. In short, the code is tail-recursive, but the manner in which one reasons about it is recursive.

Minamide [22] proposes a notion of "data structure with a hole", or in other words, a segment, and applies it to the problem of concatenating immutable lists. Walker and Morrisett [35] offer a tailrecursive version of mutable list concatenation in a low-level typed intermediate language, as opposed to a surface language. The manner in which they avoid reasoning about list segments is analogous to ours. There, because the code is formulated in continuationpassing style, the reasoning step that takes place "after the recursive call" amounts to composing the current continuation with a coercion. Maeda et al. [21] study a slightly different approach, also in the setting of a typed intermediate language, where separating implication offers a way of defining list segments. Our approach could be adapted to an iterative setting by adopting a new proof rule for while loops. This is noted independently by Charguéraud 9 . \$3.3.2] and by Tuerk [34].

\section{Syntax}

\subsection{Types}

We work with the "internal syntax" of types. The surface syntax adds a few syntactic conventions, which we explain later on $(\$ 6$. For the moment, the reader may ignore the two underlined constructs in Figure 2

Types have kinds. The base kinds are type, term, and perm. The standard types, such as function types, tuple types, etc. have kind type. The types of kind term are program variables. If a variable $x$ is bound (by let, fun, or match) in the code, then $x$ may appear not only in the code, but also in a type: it is a type of kind term. The types of kind perm are permissions. First-order arrow kinds are used to classify parameterized algebraic data types.

In Figure 2, we use the meta-variables $T$ and $X$ to stand for types and variables of arbitrary kind; we use $t$ and $P$ to suggest that a type has kind type and perm, respectively; we use $a$ and $x$ to suggest that a variable has kind type and term, respectively. We omit the definition of the kinding judgment; it appears in the

\begin{tabular}{|c|c|c|}
\hline$e::=$ & $\begin{array}{l}x \\
\text { let } p=e \text { in } e \\
\text { fun }[\vec{X}: \vec{\kappa}](x: t): t=e \\
e[t: \kappa] \\
e e \\
(\vec{e}) \\
\text { A }\{\vec{f}=\vec{e}\} \\
e . f \\
e . f \leftarrow e \\
\text { match } e \text { with } \vec{p} \rightarrow \vec{e} \\
\text { tag of } e \leftarrow \mathrm{A} \\
\text { give } e \text { to } e \\
\text { take } e \text { from } e \\
\text { fail }\end{array}$ & $\begin{array}{r}\text { expression } \\
\text { variable } \\
\text { local definition } \\
\text { anonymous function } \\
\text { type instantiation } \\
\text { function application } \\
\text { tuple } \\
\text { data constructor application } \\
\text { field access } \\
\text { field update } \\
\text { case analysis } \\
\text { tag update } \\
\text { adoption } \\
\text { abandon } \\
\text { dynamic failure }\end{array}$ \\
\hline$p::=$ & $\begin{array}{l}x \\
(\vec{p}) \\
\mathrm{A}\{\vec{f}=\vec{p}\}\end{array}$ & $\begin{array}{r}\text { pattern } \\
\text { variable } \\
\text { tuple pattern } \\
\text { data constructor pattern }\end{array}$ \\
\hline
\end{tabular}
extended version of this paper [28].

The structural type $\mathrm{A}\{\vec{f}: \vec{t}\}$ describes a block in the heap whose tag is currently $\mathrm{A}$ and whose fields $\vec{f}$ currently have the

$$
\begin{aligned}
& \kappa::=\quad \text { type } \mid \text { term } \mid \text { perm } \mid \kappa \rightarrow \kappa \quad \text { kind } \\
& T, t, P::=\quad \text { type or permission } \\
& X \quad \text { variable }(a, x, \ldots) \\
& t \rightarrow t \quad \text { function type } \\
& (\vec{t}) \quad \text { tuple type } \\
& \text { A }\{\vec{f}: \vec{t}\} \text { adopts } t \quad \text { structural type } \\
& T \vec{T} \quad n \text {-ary type application } \\
& \forall(X: \kappa) T \quad \text { universal quantification } \\
& \exists(X: \kappa) T \quad \text { existential quantification } \\
& =x \quad \text { singleton type } \\
& (t \mid P) \quad \text { type/permission conjunction } \\
& \text { dynamic (see \$7) } \\
& x @ t \quad \text { atomic permission } \\
& \text { empty empty permission } \\
& P * P \quad \text { permission conjunction } \\
& x: t \quad \text { name introduction (see } \$ 6 \text { ) }
\end{aligned}
$$

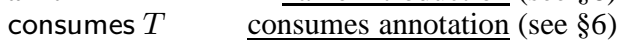

$$
\begin{aligned}
& d::=\quad \text { algebraic data type definition } \\
& \text { mutable? data } d(\vec{X}: \vec{\kappa})=\vec{b} \\
& \text { adopts } t \\
& b::=\mathrm{A}\{\vec{f}: \vec{t}\} \quad \text { algebraic data type branch }
\end{aligned}
$$

Figure 2. Syntax of types and permissions

Figure 3. Syntax of expressions

types $\vec{t}$. An example, taken from $\$ 2$ is MCons $\{$ head : $a$; tail : ()\}. The data constructor $A$ must refer to a previously defined algebraic data type, and the fields $\vec{f}$ must match the definition of $\mathrm{A}$. The types $\vec{t}$, however, need not match the types that appear in the definition of A. For instance, in the definition of MCons, the type of the tail field is mlist $a$, not (). This implies that the above structural type cannot be folded to mlist $a$; the tail field must be updated first. A structural type may include a clause of the form adopts $t$, whose meaning is explained later on (\$7). If omitted, adopts $\perp$ is the default. 
An example of a type application $T \vec{T}$ is list int. We sometimes refer to this as a nominal type, as opposed to a structural type.

The universal and existential types are in the style of System $F$. A (base) kind annotation is mandatory; if omitted, type is the default. The bottom type $\perp$ and the top type unknown can be defined as $\forall a . a$ and $\exists a . a$, respectively.

The conjunction of a type and a permission is written $(t \mid P)$. Because permissions do not exist at runtime, a value of this type is represented at runtime as a value of type $t$. Such a conjunction is typically used to express function pre- and postconditions. The type $(() \mid P)$ is abbreviated as $(\mid P)$.

Algebraic data type definitions are prefixed with the keyword data. They are anologous to Haskell's and OCaml's. Each branch is explicitly named by a data constructor and carries a number of named fields. If a definition begins with the keyword mutable, then the tag and all fields are considered mutable, and can be modified via tag update and field update instructions; otherwise, they are considered immutable. Examples appear at the top of Figure1 Like a structural type, an algebraic data type definition may include an adopts clause; if omitted, adopts $\perp$ is the default.

\subsection{Expressions}

Expressions (Figure 3) form a fairly standard $\lambda$-calculus with tuples and algebraic data structures. A function definition must be explicitly annotated with the function's type parameters, argument type, and return type. One reason for this is that the argument and return type serve as pre- and postconditions and in general cannot be inferred. Furthermore, we have System $F$-style polymorphism. Explicit type abstractions are built into function definitions. Type applications must in principle be explicit as well. The prototype type-checker allows omitting them and performs a limited form of type inference, which is outside the scope of this paper.

\section{Ownership, modes, and extent}

We wrote earlier $(\$ 1$ that "to have a permission for $x$ " can be understood informally as "to own $x$ ". Roughly speaking, this is true, but we must be more precise, for two reasons. First, we wish to distinguish between mutable data, on which we impose a "unique owner" policy, and immutable data, for which there is no such restriction. For this reason, types and permissions come in several flavors, which we refer to as modes (\$4.1). Second, in a permission of the form $x @ t$, the type $t$ describes the extent to which we own $x$. If $x s$ is a list cell, do we own just this cell? the entire spine? the spine and the elements? The answer is given by the type $t$. For instance $(\$ 4.2), x s @$ Cons $\{$ head $=h d$; tail $=t l\}$ represents the ownership of just the cell $x s$, because the singleton types $=h d$ and $=t l$ denote the ownership of an empty heap fragment. On the other hand, $x s @$ Cons $\{$ head : $a$; tail : list $a\}$ gives access to the entire list spine. (Because list is an immutable algebraic data type, this is read-only, shared access.) It further gives access to all of the list elements, insofar as the type $a$ allows this access. In this example, $a$ is a variable: one must wait until $a$ is instantiated to determine what the elements are and to what extent we own them.

\subsection{Modes}

A subset of the permissions are considered duplicable, which means that they can be implicitly copied (DUPLICATE Figure 6. Copying a permission for an object $x$ means that $x$ may be shared: it may be used via different pointers, or by different threads simultaneously. Thus, a duplicable permission does not represent unique ownership; instead, it denotes shared knowledge. Because the system does not control with whom this knowledge is shared, this knowledge must never be invalidated, lest some principals be left with an outdated version of the permission. Therefore, a duplicable permission denotes shared, permanent knowledge. The permissions that describe read-only, immutable data are duplicable: for instance, $x s @$ Cons $\{$ head $=h d$; tail $=t l\}$ and $x s @$ list int are duplicable.

A subset of the permissions are considered exclusive. An exclusive permission for an object $x$ represents the "unique ownership" of $x$. In other words, such a permission grants read-write access to the memory block at address $x$ and guarantees that no-one else has access to this block. The permissions that describe mutable memory blocks are exclusive: for instance, $x s @$ MCons $\{$ head $=$ $h d$; tail $=t l\}$ is exclusive. An exclusive permission is analogous to a "unique" permission in other systems [5] and to a separation logic assertion [29].

By lack of space, we must unfortunately omit the definition of the predicates " $t$ is duplicable" and " $t$ is exclusive", which are used in the typing rules (Figure 44). They can be found in the extended version of this paper.

No permission is duplicable and exclusive. Some permissions are neither duplicable nor exclusive. " $x s @$ list (ref int)", which describes an immutable list of references to integers, illustrates this. It must not be duplicated: this would violate the "unique owner" property of the list elements. It is not exclusive: the list cell at xs is an immutable object, and this permission does not guarantee exclusive access to this cell. Another example is " $x$ \& a". Because a is a type variable, one cannot assume that this permission is duplicable (or exclusive)

Every permission is affine. One can implicitly drop a permission that one does not need.

The language is designed so that the type-checker (and the programmer!) can always tell what mode a permission $P$ satisfies: duplicable, exclusive, or neither (hence, affine). Modes form an upper semi-lattice, whose top element is "affine", and where "duplicable" and "exclusive" are incomparable. Because algebraic data types are recursively defined, their mode analysis requires a fixed point computation, whose details we omit.

If $t$ and $u$ are exclusive types, then the conjunction $x @ t * y @ u$ implies that $x$ and $y$ are distinct addresses. In other words, conjunction of exclusive permissions is separating. On the other hand, if $t$ and/or $u$ are duplicable, $x$ and $y$ may be aliases. Conjunction is not in general separating. Conjunction of duplicable permissions requires agreement between the two conjuncts. The reader is referred to the draft paper that accompanies the type soundness proof [26] for a formal definition of the semantics of conjunction.

\subsection{Extent}

Every type $t$ has an ownership reading: that is, the permission $x @ t$ represents certain access rights about $x$. However, the extent of these rights (or, in separation logic terminology, their footprint) depends on the type $t$.

A singleton type $=y$, for instance, has empty extent. Indeed, the permission $x @=y$, which we usually write $x=y$, asserts that $x$ and $y$ are equal, but does not allow assuming that $x$ is a pointer, let alone dereferencing it.

A structural type such as Cons $\{$ head $=h d$; tail $=t l\}$ has an extent of one memory block. The permission $x s @$ Cons $\{$ head $=$ $h d$; tail $=t l\}$ gives us (read-only, shared) access to the block at address $x s$, and guarantees that its head and tail fields contain the values $h d$ and $t l$, respectively, but (as per the semantics of singleton types) guarantees nothing about $h d$ and $t l$.

What is the extent of a "deep" composite type, such as the structural type Cons $\{$ head : $a$; tail : list $a\}$ or the nominal type list $a$ ? What does it mean to own a list? In order to answer these

\footnotetext{
${ }^{3}$ Mezzo allows the programmer to explicitly assume that a type variable a is duplicable, or exclusive. This mechanism is not treated in this paper.
} 
questions, one must understand how a composite permission is decomposed into a conjunction of more elementary permissions.

A structural permission, such as $x s @$ Cons $\{$ head : $a$; tail : list $a\}$, can be decomposed by introducing a fresh name for each of the values stored in the fields. (See DECOMPOSEBLOCK in Figure 6) The result is a more verbose, but logically equivalent, permission:

$\exists h d, t l .(x s @$ Cons $\{$ head $=h d ;$ tail $=t l\} * h d @ a * t l @$ list $a)$

The meaning and extent of the original structural permission is now clearer: it grants access to the cell at $x s$ and to the first list element (to the extent dictated by the type $a$ ) and to the rest of the list.

The meaning of a nominal permission, such as $x s @$ list $a$, is just the disjunction of the meanings of its unfoldings, namely $x s @$ Nil and $x s @$ Cons $\{$ head : $a$; tail : list $a\}$.

If $a$ is (instantiated with) an exclusive type, then we find that $x s @$ list $a$ implies that the list elements are pairwise distinct, and grants read-only access to the list spine and exclusive access to the list elements.

\section{Type assignment}

\subsection{The typing judgment}

The typing judgment takes the form $K ; P \vdash e: t$. It is inductively defined in Figures 4 and 5 The kind environment $K$ maps variables to kinds. This judgment means that, by consuming the permission $P$, the expression $e$ produces a value of type $t$. It is analogous to a Hoare logic or separation logic triple, where $P$ is the precondition and $t$ is the postcondition.

The typing judgement relies on a well-kindedness judgement of the form $K \vdash t: \kappa$. It ensures that types are well-kinded and that the syntactic facilities of the surface syntax $(\$ 6$ are used properly. For conciseness, in the typing rules, we omit all freshness and wellkindedness side conditions.

The typing rules require many sub-expressions to be variables. For instance, the rule READ cannot handle a field access expression of the form $e . f$ : instead, it requires $x . f$. This requirement is met by first performing a monadic transformation, which introduces extra let constructs. Furthermore, the pattern matching rules (Figure 5 cannot handle deep patterns: they require shallow patterns. Again, this requirement is met by introducing extra let constructs. We omit the details of these transformations.

VAR is the axiom rule. It is worth noting that, in conjunction with the subsumption rule EQUALITYREFLEXIVE (Figure 6), it allows proving that $x$ has type $=x$, even in the absence of any hypothesis about $x$.

LET corresponds to the sequence rule of separation logic.

FUNCTION states that a duplicable permission $P$ that exists at the function definition site is also available within the function body. Requiring $P$ to be duplicable allows us to consider every function type duplicable. Thus, a function can be shared without restriction and can be invoked as many times as desired, provided of course that one is able to satisfy its precondition. If one wishes to write a function that captures a non-duplicable permission $P$, and can be invoked at most once, this is still possible. Indeed, a type $t_{1} \multimap t_{2}$ of "one-shot" functions can be defined as:

$$
\exists(p: \text { perm })\left(\left(\left(t_{1} \mid p\right) \rightarrow t_{2}\right) \mid p\right)
$$

This is a conjunction of a function whose precondition is $p$ and of one copy of $p$. Because $p$ is abstract, it is considered affine. Hence, at most one call is possible, after which $p$ is consumed and the function becomes unusable.

APPLICATION corresponds to the rule for procedure calls in separation logic. The caller gives up the permission $x_{2} @ t_{2}$, which is consumed, and in return gains a permission for the result of the function call, at type $t_{1}$. In other words, because types have an own- ership reading, a function type $t_{1} \rightarrow t_{2}$ describes not only the shape of the function's arguments and results, but also the side effects that the function may perform, as well as the transfers of ownership that occur from the caller to the callee and back.

NEW uses a structural type to describe the newly-allocated memory block in an exact manner. TUPLE is analogous.

READrequires a structural permission $x @ \mathrm{~A}\{F[f: t]\}$, which guarantees that $x$ points to a memory block that contains a field named $f$, and allows us to dereference $x$. $f$. READ concludes that the field access expression $x . f$ has type $t$, and that the structural permission $x @ \mathrm{~A}\{F[f: t]\}$ is preserved. There is a catch: because the type $t$ occurs twice in this postcondition, we must require $t$ to be duplicable, or the rule would be unsound. Fortunately, this is not a problem: by using DECOMPOSEBLOCK (Figure 6, also explained earlier, see $\$ 2.2$ and $\$ 4.2$, it is possible to arrange for $t$ to be a singleton type, which is duplicable.

Like READ, WRITE requires a structural permission, of the form $x_{1} @ \mathrm{~A}\left\{F\left[f: t_{1}\right]\right\}$. It checks that this permission is exclusive, i.e., the data constructor $A$ is associated with a mutable algebraic data type. This ensures that we have write access. In fact, since we have exclusive access to $x_{1}$, a strong (type-changing) update is sound. The permission is changed to $x_{1} @ \mathrm{~A}\left\{F\left[f: t_{2}\right]\right\}$, where $t_{2}$ is the type of $x_{2}$. Without loss of generality, one may let $t_{2}$ be the singleton type $=x_{2}$. This allows the type-checker to record that $x_{1} . f$ and $x_{2}$ are now aliases. If desired, the permissions $x_{1} @ \mathrm{~A}\{F[f=$ $\left.\left.x_{2}\right]\right\}$ and $x_{2} @ t_{2}$ can later be combined by DECOMPOSEBLOCK to yield $x_{1} @ \mathrm{~A}\left\{F\left[f: t_{2}\right]\right\}$. Because DECOMPOSEBLOCK read from right to left, involves a loss of information, it is typically applied by the type-checker only "on demand", i.e., to satisfy a function postcondition or a type annotation.

MATCH is used to type-check a case analysis construct. Each branch is type-checked independently. We currently do not check that the case analysis is exhaustive, but plan to add this feature in the future. The premise relies on a judgment of the form $K ; P \vdash$ let $p=x$ in $e: t$. This is not a new judgment; it is an ordinary typing judgement, but, for clarity, the typing rules that have a conclusion of this form are isolated in Figure 5 Although these rules may appear somewhat daunting, they are in fact quite straightforward. LETTUPLE checks that $x$ is a tuple, i.e., we have a permission of the form $x @\left(t_{1}, \ldots, t_{n}\right)$. If that is the case, then matching $x$ against the tuple pattern $\left(x_{1}, \ldots, x_{n}\right)$ is permitted, and gives rise to a conjunction of permissions of the form $x_{i} @ t_{i}$. Because the permission for $x$ is not lost, the types $t_{i}$ are duplicated, so they are required to be duplicable. Again, this requirement causes no loss of generality, since one can arrange to introduce singleton types ahead of time. LETDATAMATCH is analogous to LETTUPLE, but concerns a (mutable or immutable) memory block. LETDATAMISMATCH concerns the situation where the pattern, which mentions the data constructor $\mathrm{B}$, will clearly not match $x$, which is statically known to have the tag A. In that case, the branch is dead code, and is considered well-typed. LETDATAUNFOLD refines a nominal permission, such as $x @$ list $a$, by replacing it with a structural one, such as $x @$ Cons $\{$ head : $a$; tail : list $a\}$, obtained by unfolding the algebraic data type and specializing it with respect to the data constructor that appears in the pattern. We omit the exact definition of unfolding.

WRITETAG type-checks a tag update instruction, which modifies the tag carried by a memory block. Like WRITE, it requires an exclusive permission for this block. It further requires the new tag $B$ to carry the same number of fields as the previous tag $A$.

\footnotetext{
${ }^{4}$ We write $F[f: t]$ for a sequence of field/type pairs in which the pair $f: t$ occurs. The adopts clause, if present, is irrelevant. We overload field names: there could exist multiple data constructors with a field named $f$. There can be at most one permission of the form $x @ \mathrm{~A}\{F[f: t]\}$, though, which allows disambiguation to take place.
} 


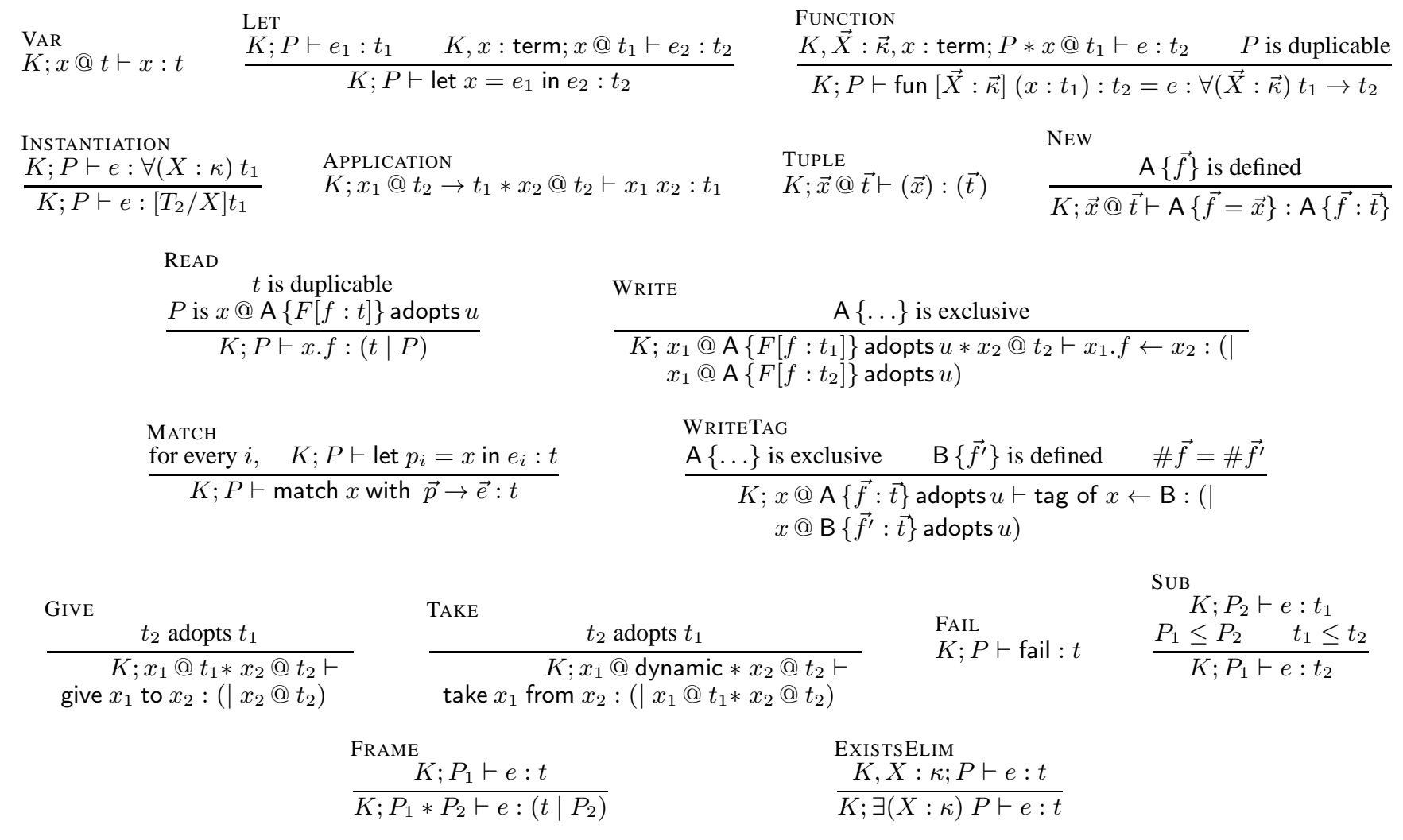

Figure 4. Typing rules

LETTUPLE

$(\vec{t})$ is duplicable

$\frac{K, \vec{x}: \text { term; } P * x @(\vec{t}) * \vec{x} @ \vec{t} \vdash e: t}{K ; P * x @(\vec{t}) \vdash \operatorname{let}(\vec{x})=x \text { in } e: t}$

LETDATAMismatCh

$\mathrm{A}$ and $\mathrm{B}$ belong to a common algebraic data type

$\overrightarrow{K ; P * x @ \mathrm{~A}\{\vec{f}: \vec{t}\} \text { adopts } u \vdash \text { let } \mathrm{B}\left\{\overrightarrow{f^{\prime}}=\vec{x}\right\}=x \text { in } e: t}$
LETDATAMATCH

$$
\begin{aligned}
& \frac{K, \vec{x}: \text { term; } P * x @ \mathrm{~A}\{\vec{f}: \vec{t}\} \text { adopts } u * \vec{x} @ \vec{t} \vdash e: t}{K ; P * x @ \mathrm{~A}\{\vec{f}: \vec{t}\} \text { adopts } u \vdash \text { let } \mathrm{A}\{\vec{f}=\vec{x}\}=x \text { in } e: t} \\
& \text { LetDataUnfold } \\
& x @ \mathrm{~A}\{\vec{f}: \vec{t}\} \text { adopts } u \text { is an unfolding of } T \vec{T} \\
& \frac{K ; P * x @ \mathrm{~A}\{\vec{f}: \vec{t}\} \text { adopts } u \vdash \text { let } \mathrm{A}\{\vec{f}=\vec{x}\}=x \text { in } e: t}{K ; P * x @ T \vec{T} \vdash \text { let } \mathrm{A}\{\vec{f}=\vec{x}\}=x \text { in } e: t}
\end{aligned}
$$

\begin{tabular}{|c|c|c|c|c|c|}
\hline \multicolumn{2}{|c|}{$\begin{array}{l}\text { EQUALITYREFLEXIVE } \\
\text { empty } \leq(x=x)\end{array}$} & \multicolumn{2}{|c|}{$\begin{array}{l}\text { EQUALSFOREQUALS } \\
\left(y_{1}=y_{2}\right) *\left[y_{1} / x\right] P \equiv\left(y_{1}=y_{2}\right) *\left[y_{2} / x\right] P\end{array}$} & $\frac{P \text { DuPLICATE }}{P \text { is duplicable }}$ & $\begin{array}{l}\text { MixSTAR } \\
x @ t * P \equiv x @(t \mid P)\end{array}$ \\
\hline $\begin{array}{l}\text { WEAKEN } \\
P_{1} * P_{2} \leq P_{2}\end{array}$ & $\begin{array}{l}\text { ExIST } \\
{[T / X}\end{array}$ & $\leq \exists(X: \kappa) P$ & $\begin{array}{l}\text { Exists ATOMIC } \\
\quad x @ \exists(X: \kappa) t \\
\equiv \exists(X: \kappa)(x @ t)\end{array}$ & \multicolumn{2}{|c|}{$\begin{array}{l}\text { DecomposeBlock } \\
y \text { @ A }\{F[f: t]\} \text { adopts } u \\
\equiv \exists(x: \text { term })(y @ \mathrm{~A}\{F[f=x]\} \text { adopts } u * x @ t)\end{array}$} \\
\hline \multicolumn{3}{|c|}{$\begin{array}{l}\text { FOLD } \\
\text { A }\{\vec{f}: \vec{t}\} \text { adopts } u \text { is an unfolding of } T \vec{T}\end{array}$} & \multicolumn{2}{|c|}{$\begin{array}{c}\text { A }\{\vec{f}: \vec{t}\} \text { adopts } u \text { is an unfolding of } T \vec{T} \\
T \vec{T} \text { has only one branch }\end{array}$} & $\begin{array}{l}\text { DYNAMICAPPEARS } \\
t \text { is exclusive }\end{array}$ \\
\hline \multicolumn{3}{|c|}{$x @ \mathrm{~A}\{\vec{f}: \vec{t}\}$ adopts $u \leq x @ T \vec{T}$} & $x @ T \vec{T} \leq x @ \mathrm{~A}\{\vec{f}$ & $\overrightarrow{\vec{t}\} \text { adopts } u}$ & $\overline{x @ t \leq x @ t * x @ \text { dynamic }}$ \\
\hline
\end{tabular}

Figure 5. Auxiliary typing rules for pattern matching

Figure 6. Permission subsumption (not all rules shown) 
(Thus, the block does not have to be enlarged or shrunk.) The structural permission is updated in a straightforward way. The types $\vec{t}$ of the fields do not change. The names of the fields change from $\vec{f}$ to $\overrightarrow{f^{\prime}}$, where the sequences of fields are ordered in the same way as in the (user-provided) definitions of $A$ and $B$. It is worth noting that $A$ and $B$ need not belong to the same algebraic data type: thus, a memory block can be re-used for a completely new purpose. Furthermore, the tag B may be associated with an immutable algebraic data type: in that case, the block is frozen, that is, becomes forever immutable. This feature is exploited in the concatenation of immutable lists (Figure 1, line 19).

GIVE and TAKE are explained later on ( $\$ 7$ ).

SUB is analogous to Hoare's rule of consequence. It relies on permission subsumption, $P_{1} \leq P_{2}$, defined in Figure 6 and discussed further on $\left(\$ 5.2\right.$, and on subtyping, $t_{1} \leq t_{2}$, defined as $x @ t_{1} \leq x @ t_{2}$ for a fresh $x$.

FRAME is analogous to the frame rule of separation logic.

\subsection{The permission subsumption judgment}

A subse 5 of the subsumption rules appears in Figure 6 Since $x=y$ is sugar for $x @=y$, the rule EQUALITYREFLEXIVE can be understood as a claim that $x$ inhabits the singleton type $=x$. EQUALSFOREQUALS shows how equations are exploited: if $y_{1}$ and $y_{2}$ are known to be equal, then they are interchangeable. (We write $\equiv$ for subsumption in both directions.) DUPLICATE states that a permission that is syntactically considered duplicable can in fact be duplicated. MIXSTAR introduces and eliminates $(t \mid P)$. WEAKEN states that every permission is affine. EXISTSINTRO introduces an existential permission; EXISTSATOMIC converts between an existential permission and an existential type. When read from left to right, DECOMPOSEBLOCK which was discussed earlier ( $\$ 2.2$ $\$ 4.2$, introduces a fresh name $x$ for the value stored in $y . f$. When read from right to left, it forgets such a name. (In that case, it is typically used in conjunction with EXISTSINTRO) This rule is related to Sing\#'s explicit "expose" [15]. FoLD folds an algebraic data type definition, turning a structural type into a nominal type. Unfolding is normally performed by case analysis (see LETDATAUNFOLD in Figure 5), but in the special case where an algebraic data type has only one branch (i.e., it is a record type), it can be implicitly unfolded by UNFOLD DYNAMICAPPEARS is explained later on (\$7).

\subsection{The Mezzo type-checker}

Implementing a checker for a highly non-deterministic system such as Mezzo poses technical challenges. Our type-checker greedily introduces fresh auxiliary names so as to "normalize" the types and permissions at hand. A persistent union-find data structure keeps track of the permissions of the form " $x=y$ ". The use of flexible variables enables a limited form of type inference. At present, the implemented type-checker is not complete with respect to the type assignment rules. This is only a brief overview: a follow-up paper devoted to the implementation of Mezzo is in the works.

\section{Surface syntax}

The internal syntax, which we have been using so far, can be fairly verbose. To remedy this, we introduce two syntactic conventions, which rely on the name introduction construct and on the consumes keyword (Figure 2). Two transformations eliminate these constructs, so as to obtain a type expressed in the internal syntax. They are formalized in the extended version of the present paper [28]. Here, we give only an intuition.

\footnotetext{
${ }^{5}$ The reader is referred to the extended version of this paper [28] for the full set of rules. The omitted rules include: conjunction is commutative, associative, and has unit empty; covariance and contravariance of the type constructors; and a few more.
}

\subsection{The name introduction form}

The construct $x: t$ allows introducing a name $x$ for a component of type $t$. This allows writing "dependent function types", such as $\left(x_{1}: t_{1}\right) \rightarrow\left(x_{2}: t_{2}\right)$, where by convention $x_{1}$ is bound within $t_{1}$ and $t_{2}$, while $x_{2}$ is bound within $t_{2}$. This is desugared by quantifiying $x_{1}$ universally above the arrow and quantifying $x_{2}$ existentially in the right-hand side of the arrow.

As an example, consider the type of :=, the function that writes a reference. This function expects a pair of a reference $x$ whose content has type $a$ and of a value of type $b$, which it stores into $x$. At the end, $x$ has become a reference whose content has type $b$. The variable $x$ must be mentioned in the pre- and postcondition. In the internal syntax, the type of $:=$ is:

$$
\forall a, b . \forall(x: \text { term })((=x \mid x @ \operatorname{ref} a), b) \rightarrow(\mid x @ \operatorname{ref} b)
$$

Thanks to the name introduction form, instead of planning ahead and quantifying $x$ in front of the function type, one names the first argument " $x$ " on the fly. Thus, in the surface syntax, one writes:

$$
\forall a, b \text {. (consumes } x: \text { ref } a \text {, consumes } b) \rightarrow(\mid x @ \operatorname{ref} b)
$$

This is not significantly shorter, because of the consumes keyword, which must be used in the surface syntax, as explained below. In actual use, though, the comfort afforded by this feature is critical.

\subsection{The consumes annotation}

Often, a permission is required and returned by a function, in which case it is unpleasant to have to write this permission twice, in the precondition and postcondition. Drawing inspiration from Sing\# [15], we adopt the convention that, in the surface syntax, by default, the permission for the argument is required and returned, i.e., it is not consumed.

For instance, the type of the list length function, which in the internal syntax is:

$$
\forall a . \forall(x: \text { term })(=x \mid x @ \text { list } a) \rightarrow(\text { int } \mid x @ \text { list } a)
$$

can in the surface syntax be written in a much more pleasant form:

$$
\forall a \text {.list } a \rightarrow \text { int }
$$

The type list $a$ is mentioned once, instead of twice, and as a side effect, the need to name the argument $x$ vanishes.

When a permission is consumed, though, we need a way of indicating this. This is the purpose of the consumes keyword. When a component is marked with this keyword, the permission for this component is required and not returned. This keyword makes sense only in the left-hand side of an arrow.

Since internal syntax and surface syntax interpret the function type differently, a translation is required, regardless of whether consumes is used. Consider a function type of the form $t \rightarrow u$, where $t$ does not contain any name introduction forms. Let $t_{1}$ stand for $[\tau /$ consumes $\tau] t$, i.e., a copy of $t$ where the consumes keyword is erased. Let $t_{2}$ stand for [ $T /$ consumes $\left.\tau\right] t$, i.e., a copy of $t$ where every component marked with this keyword is replaced with $T^{6}$. Then, the translation of this function type is $\left(x: t_{1}\right) \rightarrow(u)$ $\left.x @ t_{2}\right)$. The parts of the argument that are not marked as consumed are returned to the caller.

The type of the function insert, which appears in Figure 7 and is discussed in $\$ 7.1$ states that the first argument is consumed, while the second argument is not. Its translation into the internal syntax is as follows:

$$
\begin{aligned}
& \forall(a: \text { type }) \forall(x: \text { term }) \\
& (=x \mid x @(a, \text { bag } a)) \rightarrow(\mid x @(\text { unknown, bag } a))
\end{aligned}
$$

\footnotetext{
6 Here, we write $T$ for unknown or empty, depending on whether the consumes keyword is applied to a type or a permission.
} 


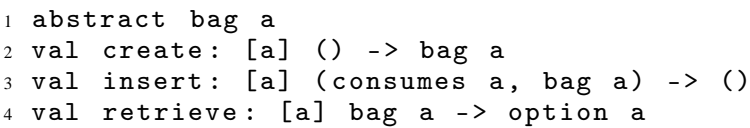

Figure 7. An interface for bags

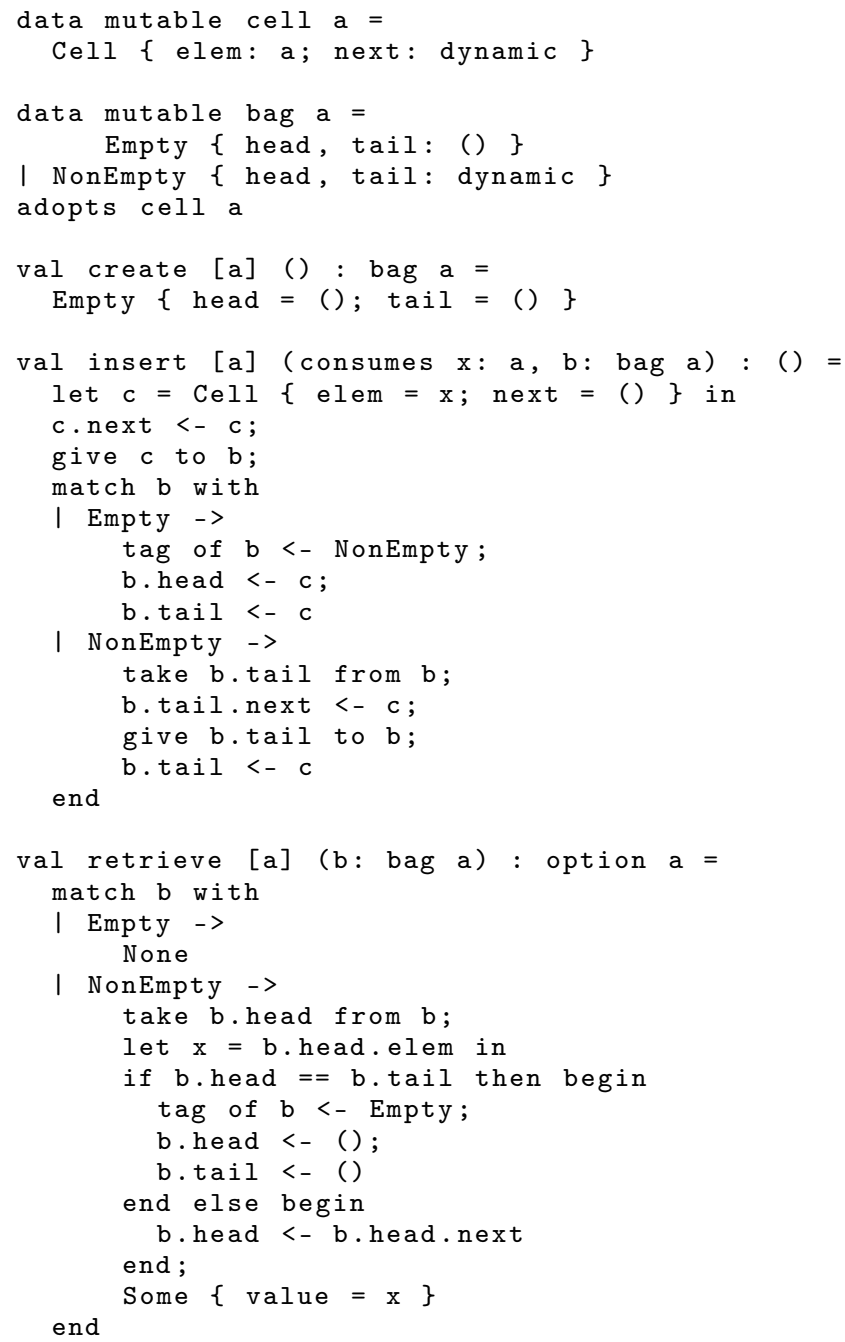

Figure 8. A FIFO implementation of bags

\subsection{Function definitions}

In the internal syntax, functions take the form fun $\left(x: t_{1}\right): t_{2}=e$, where one variable, namely $x$, is bound in $e$. In the surface syntax, instead, functions take the form fun $t_{1}: t_{2}=e$. The argument type $t_{1}$ is interpreted as a pattern, and the names that it introduces are considered bound in $e$. An example is fun ( $x:$ int, $y:$ int) : int $=x+y$, where $(x:$ int, $y:$ int $)$ is the type of the argument, int is the type of the result, and $x$ and $y$ are bound in the function body, which is $x+y$.

\section{Adoption and abandon}

The permission discipline that we have presented so far has limited expressive power. It can describe immutable data structures with ar- bitrary sharing and tree-shaped mutable data structures. However, because mutable memory blocks are controlled by exclusive permissions, it cannot describe mutable data structures with sharing.

\subsection{Overview}

In order to illustrate this problem, let us imagine how one could implement a "bag" abstraction. A bag is a mutable container, which supports two operations: inserting a new element and retrieving an arbitrary element.

We would like our implementation to offer the interface in Figure 7 There, bag is presented as an abstract type. Because it is not explicitly declared duplicable, it is regarded as affine. Hence, a bag "has a unique owner", i.e., is governed by a nonduplicable permission. The function create creates a new bag, whose ownership is transferred to the caller. The type of insert indicates that insert $(x, b)$ requires the permissions " $x \notin t$ " and "b @ bag t", for some type $t$, and returns only the latter. Thus, the caller gives up the ownership of $\mathrm{x}$, which is "transferred to the bag". Conversely, the call "let $\circ=$ retrieve $b$ in ..." produces the permission "o @ option a", which means that the ownership of the retrieved element (if there is one) is "transferred from the bag to the caller".

To implement bags, we choose a simple data structure, namely a mutable singly-linked list. One inserts elements at the tail and extracts elements at the head, so this is a FIFO implementation. One distinguished object b, "the bag", has pointers to the head and tail of the list, so as to allow constant-time insertion and extraction. (We use "object" as a synonym for "memory block".)

This data structure is not tree-shaped: the last cell in the list is accessible via two distinct paths. In order to type-check this code, we must allow the ownership hierarchy and the structure of the heap to differ. More specifically, we would like to view the list cells as collectively owned by the bag b. That is, we wish to keep track of just one exclusive permission for the group formed by the list cells, as opposed to one permission per cell.

We use the name $b$ as a name for this group. When a cell $c$ joins the group, we say that b adopts c, and when c leaves the group, we say that $\mathrm{b}$ abandons $\mathrm{c}$. In other words, the bag $\mathrm{b}$ is an adopter, and the list cells c are its adoptees. In terms of ownership, adopter and adoptees form a unit: the exclusive permission that controls $\mathrm{b}$ also represents the ownership of the group, and is required by the adoption and abandon operations.

Adoption requires and consumes an exclusive permission for the cell c that is about to be adopted: the ownership of $c$ is transferred to the group. Conversely, abandon produces an exclusive permission for the cell $\mathrm{c}$ that is abandoned: the group relinquishes the ownership of c.

Abandon must be carefully controlled. If a cell could be abandoned twice, two permissions for it would appear, which would be unsound. Due to aliasing, though, it is difficult to statically prevent this problem. Instead, we decide to record at runtime which object is a member of which group, and to verify at runtime that abandon is used in a safe way.

\subsection{Details}

Let us now explain in detail the dynamic semantics of adoption and abandon (what these operations do) as well as their static semantics (what the type-checker requires).

Adopter fields We maintain a pointer from every adoptee to its adopter. Within every object, there is a hidden "adopter" field, which contains a pointer to the object's current adopter, if it has one, and null otherwise. This information is updated when an object is adopted or abandoned. In terms of space, the cost of this design decision is one field per object. 
The type dynamic The permission "c @ dynamic" guarantees that $\mathrm{c}$ is a pointer to a memory block and grants read access to the field c.adopter. This can be used to verify the identity of c's adopter. In other words, "c @ dynamic" can be viewed as a permission to perform a dynamic group membership test. It is a duplicable permission. It appears spontaneously when $\mathrm{c}$ is known to be a (mutable) object: this is stated by the rule DYNAMICAPPEARS in Figure 6

In the bag implementation, shown in Figure 8 , the head and tail fields of a non-empty bag object, as well as the next field of every cell object, have type dynamic (lines 2 and 6. Because dynamic is duplicable, sharing is permitted: for instance, the pointers b.head and b.tail might happen to be equal.

Adopts clauses When a cell c is adopted, the exclusive permission that describes it, namely "c @ cell a", disappears. Only "c @ dynamic" remains. As a result, the information that $c$ is a cell is lost: the type-checker can no longer tell how many fields exist in the object $\mathrm{c}$ and what they contain. When the bag b later abandons c, we would like the permission "c @ cell a" to re-appear. How can the type-checker recover this information?

Fortunately, when $\mathrm{b}$ abandons $\mathrm{c}$, the type-checker has access to the type of $b$. Thus, provided the type of the adopter determines the type of its adoptees, this problem is solved.

For an object $b$ of type $t$ to serve as an adopter, where $t$ is an algebraic data type, we require that the definition of $t$ contain the clause "adopts u" and that $t$ and $u$ be exclusive types. This is illustrated in Figure 8. where the definition of "bag a" says "adopts cell a" (line 7).

Because the type of the adoptees must not be forgotten when an algebraic data type is unfolded, structural permissions also carry an adopts clause. In the case of bags, for instance, the permission "b @ bag a" is refined by the match constructs of lines 16 and 29 into either "b @ Empty \{ head, tail: () \} adopts cell a" or "b @ NonEmpty \{ head, tail: dynamic \} adopts cell a", and, conversely, either of these permissions can be folded back to "b @ bag a".

We write that " $t$ adopts $u$ " if either $t$ is an algebraic data type whose definition contains the clause "adopts $u$ " or $t$ is a structural type that contains the clause "adopts u".

Adoption The syntax of adoption is "give c to b". This instruction requires two permissions "c @ u" and "b @ $t$ ", where $t$ adopts $u$ GIVE, Figure 4). At the program point that follows this instruction, the permission "b @ t" remains available, but "c @ u" has been consumed. Fortunately, not everything about $c$ is forgotten. The permission "c @ dynamic", which is present before the adoption instruction because "c @ u" spontaneously gives rise to "c @ dynamic", remains present after adoption.

The runtime effect of this operation is to write the address $b$ to the field c.adopter. The exclusive permission "c @ u" guarantees that this field exists and that its value, prior to adoption, is null.

In the bag implementation (Figure 8), adoption is used at the beginning of insert (line 15], after a fresh cell c has been allocated and initialized. This allows us to maintain the (unstated) invariant that every cell that is reachable from $b$ is adopted by $b$.

Abandon The syntax of abandon is "take c from b". This instruction requires "b @ t" and "c @ dynamic", where t adopts "u", for some type u (TAKE Figure 4). After this instruction, "b @ t", remains available. Furthermore, the permission "c @ u" appears.

The runtime effect of this operation is to check that the field c.adopter contains the address $\mathrm{b}$ and to write null into this field, so as to reflect the fact that b abandons c. If this check fails, the execution of the program is aborted.

In the bag implementation (Figure 8), abandon is used near the beginning of retrieve, at line 33 There, the first cell in the queue, $\mathrm{b}$.head, is abandoned by $\mathrm{b}$. This yields a permission at type "cell a" for this cell. This permission lets us read b.head.elem and $\mathrm{b}$.head.next and allows us to produce the permission " $\mathrm{x} @ \mathrm{a}$ ", where $\mathrm{x}$ is the value found in $\mathrm{b}$.head.elem.

Abandon and adoption are also used inside insert, at lines 22 and 24 There, the bag $\mathrm{b}$ is non-empty, and the cell b.tail must be updated in order to reflect the fact that it is no longer the last cell in the queue. However, we cannot just go ahead and access this cell, because the only permission that we have at this point for this cell is at type "dynamic". Instead, we must take the cell out of the group, update it, and put it back. We allow writing "taking b.tail from b begin ... end" as sugar for such a wellparenthesized use of take and give.

\subsection{Discussion}

To the best of our knowledge, adoption and abandon are new. Naturally, the concept of group, or region, has received sustained interest in the literature [11, 12, 16, 32]. Regions are usually viewed either as a dynamic memory management mechanism or as a purely static concept. Adoption and abandon, on the other hand, offer a dynamic ownership control mechanism, which complements our static permission discipline.

Adoption and abandon are a very flexible mechanism, but also a dangerous one. Because abandon involves a dynamic check, it can cause the program to encounter a fatal failure at runtime. In principle, if the programmer knows what she is doing, this should never occur. There is some danger, but that is the price to pay for a simpler static discipline. After all, the danger is effectively less than in ML or Java, where a programming error that creates an undesired alias goes completely undetected—until the program misbehaves in one way or another.

One might wonder why the type dynamic is so uninformative: it gives no clue as to the type of the adoptee or the identity of the adopter. Would it be possible to parameterize it so as to carry either information? The short answer is negative. The type dynamic is duplicable, so the information that it conveys should be stable (i.e., forever valid). However, the type of the adoptee, or the identity of the adopter, may change with time, through a combination of strong updates and give and take instructions. Thus, it would not make sense for dynamic to carry more information.

That said, we believe that adoption and abandon will often be used according to certain restricted protocols, for which more information is stable, hence can be reflected at the type level. For instance, in the bag implementation, a cell only ever has one adopter, namely a specific bag $b$. In that case, one could hope to work with a parameterized type dynamic' $b$, whose meaning would be "either this object is currently not adopted, or it is adopted by $b$ ". Ideally, dynamic' would be defined on top of dynamic in a library module, and its use would lessen the risk of confusion.

\section{Other means of permitting sharing}

Adoption and abandon is not the only way of sharing mutable data. We now describe two other mechanisms, namely nesting and locks.

\subsection{Nesting}

Nesting [7] is a mechanism by which an object $x$ adopts (so to speak) a permission $P$. It is a purely static mechanism. The act of nesting $P$ in $x$ has no runtime effect, but consumes $P$ and produces a witness, a permission which Boyland writes $P \prec x$. Because nesting is irreversible, such a witness is duplicable.

Once $P$ has been nested in $x$, whoever has exclusive ownership of $x$ may decide to temporarily recover $P$. This is done via two symmetric operations, say "focus" and "defocus", which in the presence of $P \prec x$ convert between $x @ t$ and $P *(P+x @ t)$ 
(where the type $t$ is arbitrary, but must be exclusive). The permission $P+x @ t$ means that $P$ has been "carved out" of $x$. While this is the case, $x @ t$ is temporarily lost: in order to recover it, one must give up $P$. Thus, it is impossible to simultaneously carve $t w o$ permissions out of $x$.

Nesting subsumes Fähndrich and DeLine's adoption and focus [16]. We view it as a purely static cousin of adoption and abandon. Adoption is more flexible in several important ways: it allows accessing two adoptees at the same time, and allows abandoning an object forever. Nesting has advantages over adoption and abandon: it cannot fail at runtime; it has no time or space overhead; one may nest a permission, whereas one adopts an object; and nesting is heterogeneous, i.e., an object $x$ can nest multiple distinct permissions, whereas, in the case of adoption and abandon, all adoptees of $x$ must have the same type.

Nesting can be axiomatized in Mezzo as a trusted library, whose interface appears in the extended version of this paper [28]. In principle, this requires extending the proof of type soundness; we have not done so. When applicable, nesting seems preferable to adoption; however, adoption is more widely applicable.

\subsection{Locks}

Dynamically-allocated locks in the style of concurrent separation logic [25, 18, 19, 8] are another dynamic mechanism for mediating access to a permission. A new lock, of type lock $P$, where $P$ is an arbitrary permission, is created via a function new. The functions acquire and release both take the lock as an argument; acquire produces the permission $P$, which release consumes. The type lock $P$ is duplicable, so an arbitrary number of threads can share the lock and simultaneously attempt to acquire it. Within a critical section, delimited by acquire and release, the "lock invariant" $P$ is available, whereas, outside of it, it is not. The "invariant" $P$ can in fact be broken within the critical section, provided it is restored when one reaches the end of the section.

Locks introduce a form of hidden state into the language. Because the permission $l @$ lock $P$ is duplicable, it can be captured by a closure. As a result, it becomes possible for a function to perform a side effect, even though its type does not reveal this fact (the pre- and postcondition are empty). Mezzo's modest library for memoization exploits this feature.

Locks can be used to encode "weak" (duplicable) references in the style of ML and duplicable references with affine content in the style of Alms [33], both of which support arbitrary sharing.

Locks can be axiomatized in Mezzo as a library, whose interface appears in the extended version of this paper [28]. Again, the proof of type soundness must be extended; we have begun this work. We view locks as complementary to adoption and abandon and nesting. In a typical usage scenario, a lock protects an adopter, which in turn controls a group of adoptees (or of nested permissions). This allows a group of objects to be collectively protected by a single lock. It should be noted that (we believe) adoption and abandon are sound in a concurrent setting.

\section{Related work}

The literature offers a wealth of type systems and program logics that are intended to help write correct programs in the presence of mutable, heap-allocated state. We review a few of them and contrast them with Mezzo.

Ownership Types [10] and its descendants restrict aliasing. Every object is owned by at most one other object, and an "owneras-dominator" principle is enforced: every path from a root to an object $x$ must go through $x$ 's owner. Universe Types [14] impose a slightly different principle, "owner-as-modifier". Arbitrary paths are allowed to exist in the heap, but only those that go through $x$ 's owner can be used to modify $x$. This approach is meant to support program verification, as it allows the owner to impose an object invariant. Permission systems [5, 7, 17] annotate pointers not with owners, but with permissions. The permission carried by a pointer tells how this pointer may be used (e.g. for reading and writing, only for reading, or not at all) and how other pointers to the same object (if they exist) might be used by others.

The systems mentioned so far are refinements (restrictions) of a traditional type discipline. Separation logic [29] departs from this approach and obeys a principle that we dub "owner-as-asserter". (In O'Hearn's words, "ownership is in the eye of the asserter" [25].) Objects are described by logical assertions. To assert is to own: if one knows that " $x$ is a linked list", then one may read and write the cells that form this list, and nobody else may. Whereas the previously mentioned systems combine structural descriptions (i.e., types) with owner or permission annotations, separation logic assertions are at once structural descriptions and claims of ownership.

Mezzo follows the "owner-as-asserter" principle. In the future, this should allow us to annotate permissions with logical assertions and use that as a basis for the specification and proof of Mezzo programs. A tempting research direction is to translate Mezzo into $\mathrm{F}^{\star}$ [31]. This purely functional programming language is equipped with affine values, with powerful facilities for expressing program specifications and proofs, and with a notion of proof erasure.

Although our permission discipline is partly inspired by separation logic [29], it is original in several ways. It presents itself as a type system, as opposed to a program logic. This makes it less expressive than a program logic, but more pervasive, in the sense that it can (and must) be used at every stage of a program's development, without proof obligations. It distinguishes between immutable and mutable data, supports first-class functions, and takes advantage of algebraic data types in novel ways.

As far as we know, Ownership or Universe Types cannot express uniqueness or ownership transfer. Müller and Rudich [23] extend Universe Types with these notions. They rely on the fact that each object maintains, at runtime, a pointer to its owner. The potential analogy with our adopter fields deserves further study.

The use of singleton types to keep track of equations, and the idea that pointers can be copied, whereas permissions are affine, are inspired by Alias Types [30]. Linear [1] and affine [33] type systems support strong updates and often view permissions (or "capabilities") as ordinary values, which hopefully the compiler can erase. By offering an explicit distinction between permissions and values, we guarantee that permissions are erased, and we are able to make the flow of permissions mostly implicit. Through algebraic data types and through the type constructor $(t \mid P)$, we retain the ability to tie a permission to a value, if desired.

Regions [30, 16, 1] have been widely used as a technical device that allows a type to indirectly refer to a value or set of values. In $M e z z o$, types refer to values directly. This simplifies the metatheory and the programmer's view.

Gordon et al. [17] ensure data-race freedom in an extension of C\#. They qualify types with permissions in the set immutable, isolated, writable, or readable. The first two roughly correspond to our immutable and mutable modes, whereas the last two have no Mezzo analogue. Shared (writable) references allow legacy sequential code to be considered well-typed. A salient feature is the absence of an alias analysis, which simplifies the system considerably. This comes at a cost in expressiveness: mutable global variables, as well as shared objects protected by locks, are disallowed.

Plaid [2] and Mezzo exhibit several common traits. A Plaid object does not belong to a fixed class, but can move from one "state" to another: this is related to Mezzo's tag update. Methods carry state pre- and postconditions, which are enforced via permissions [5]. Plaid is more ambitious in that states are organized in an extensible hierarchy, whereas algebraic data types are flat and closed. 


\section{Conclusion and future work}

Mezzo is a high-level functional and imperative programming language where the traditional concept of "type" is replaced with a more powerful concept of "permission". Distinguishing duplicable, exclusive, and affine permissions allows reasoning about state changes. We strive to achieve a balance between simplicity and expressiveness by marrying a static discipline of permissions and a novel dynamic form of adoption and abandon. By adding other mechanisms for controlling sharing, such as nesting and locks, we augment the expressiveness of the language and emphasize that the permission discipline is sufficiently powerful to express these notions. Mezzo is type-safe: well-typed programs cannot go wrong (but an abandon operation can fail). We have carried out a machinechecked proof of type safety [26]. In the future, we would like to extend $M e z z o$ with support for shared-memory concurrency. We believe that, beyond locks ( 8.2 , many abstractions (threads, channels, tasks, etc.) can be axiomatized so as to guarantee that welltyped code is data-race-free.

\section{References}

1] Amal Ahmed, Matthew Fluet, and Greg Morrisett. $L^{3}$ : A linear language with Fundamenta Informatica, 77(4):397-449, 2007.

[2] Jonathan Aldrich, Joshua Sunshine, Darpan Saini, and Zachary Sparks. Typestate-oriented programming In Companion to ObjectOriented Programming, Systems, Languages, and Applications (OOPSLA), pages 1015-1022, 2009.

[3] Mike Barnett, K. Rustan M. Leino, and Wolfram Schulte. The Spec\# prog In Construction and Analysis of Safe, Secure and Interoperable Smart devices (CASSIS), volume 3362 of Lecture Notes in Computer Science, pages 49-69. Springer, 2004.

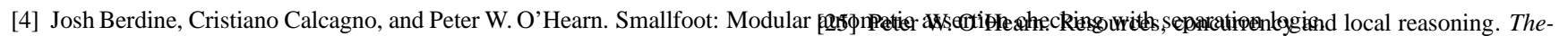
In Formal Methods for Components and Objects, volume 4111 of Lecture Notes in Computer Science, pages 115-137. Springer, 2005.

[5] Kevin Bierhoff and Jonathan Aldrich. Modular typestate checking of aliased objectslary 2013. In Object-Oriented Programming, Systems, Languages, and Applications (OOPSLA), pages 301-320, 2007.

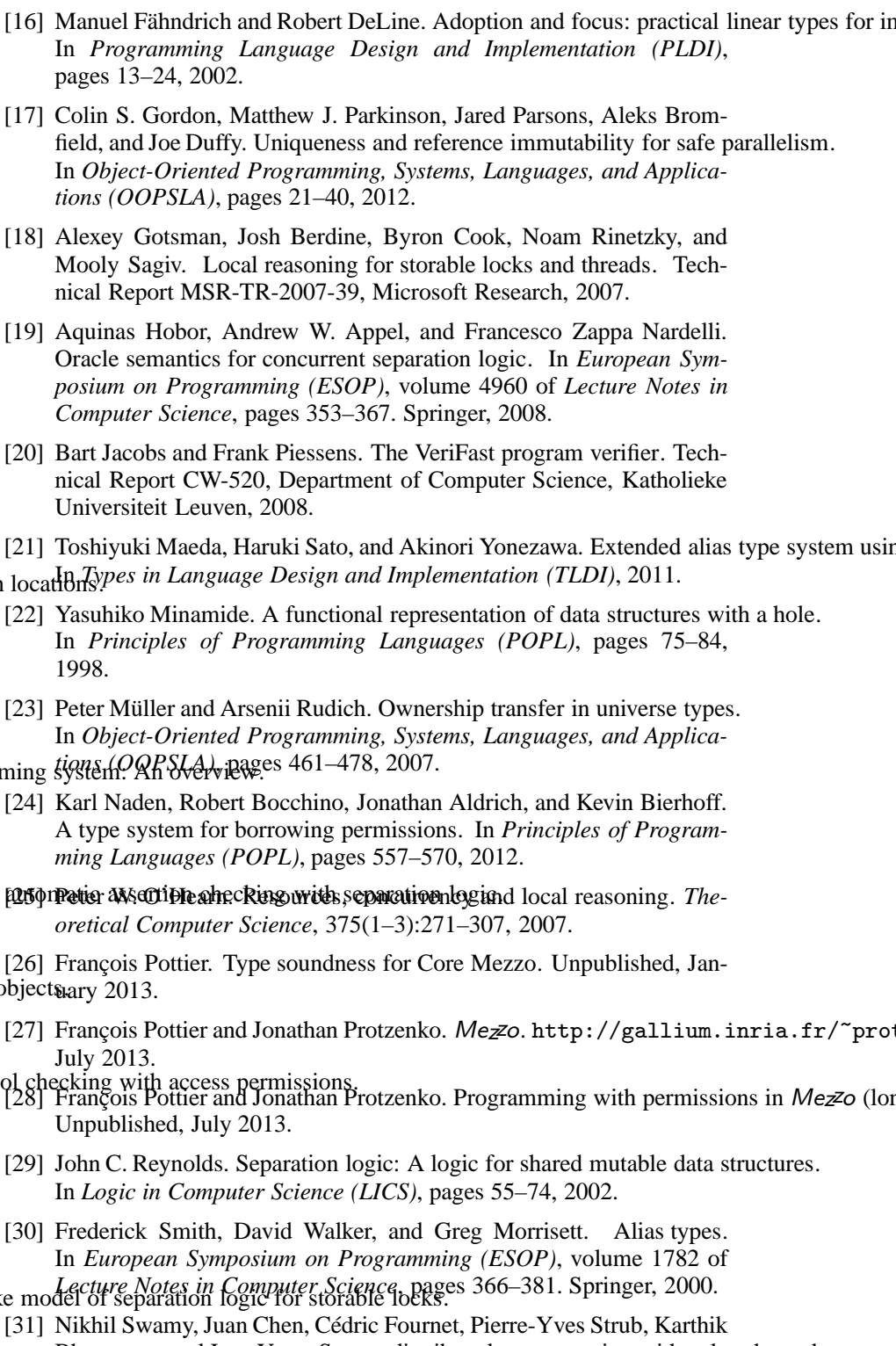

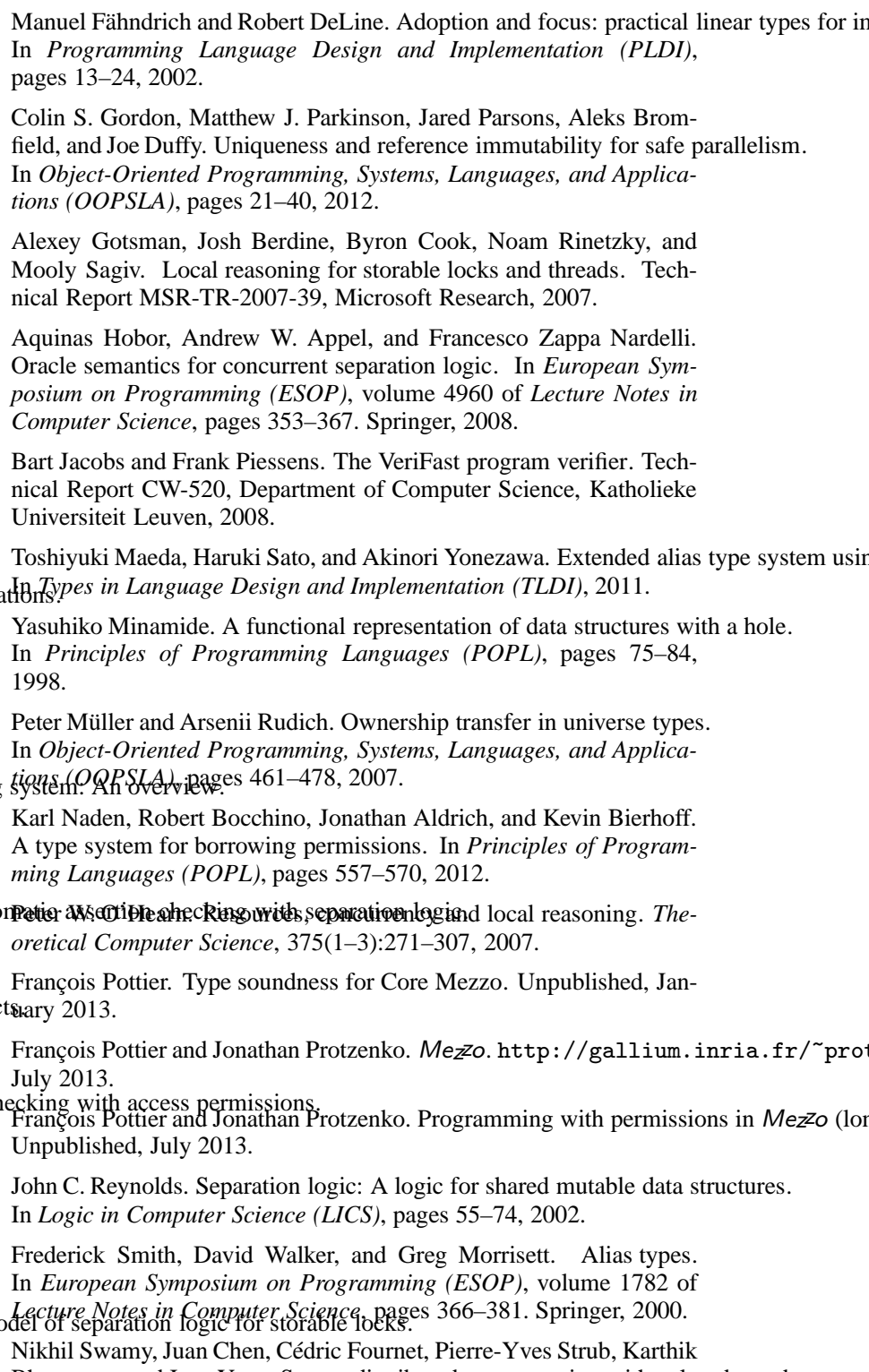

[6] Kevin Bierhoff, Nels E. Beckman, and Jonathan Aldrich. Practical API protocol checking with access permissions In European Conference on Object-Oriented Programming (ECOOP), volume 5653 of Lecture Notes in Computer Science, pages 195-219. Springer, 2009.

[7] John Tang Boyland. Semantics of fractional permissions with nesting ACM Transactions on Programming Languages and Systems, 32(6), 2010.

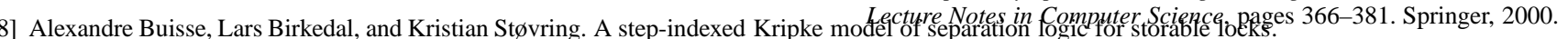
Electronic Notes in Theoretical Computer Science, 276:121-143, [31] Nikhil Swamy, Juan Chen, Cédric Fournet, Pierre-Yves Strub, Karthik 2011.

Ianuel Fähndrich, Mark Aiken, Chris Hawblitzel, Orion Hodson, In EuroSys, pages 177-190, 2006.

[16] Manuel Fähndrich and Robert DeLine. Adoption and focus: practical linear types for in to 